nucleus and control gene expression. Yet, so far, nobody has been able to detect accumulation of NICD in the nucleus in response to ligand binding in normal cells. Schroeter et al. ${ }^{1}$, working with vertebrate cells, now show that the second view is nevertheless almost certainly correct. They have identified the site at which the transmembrane Notch-1 molecule is normally cleaved to release NICD; they have demonstrated that a mutation at this site hinders Notch-1 signalling by hindering the cleavage; and they have shown that the quantities of NICD produced in response to ligand binding are enough to regulate transcription, even though they are too small to be detected by standard immunocytochemistry.

Meanwhile, two other groups (working in Drosophila) have come to a similar conclusion by a different approach. Struhl and Adachi $^{2}$, and Lecourtois and Schweisguth ${ }^{3}$, have made flies that have either a transcriptional activator domain (Gal4 or Gal4+VP16) or a repressor domain (En or WRPW) inserted into the full-length Notch protein. Such an insert is expected to regulate transcription of an appropriate reporter gene only if the fragment of Notch that contains the insert gets into the nucleus where the reporter gene lies. The regulatory effect on the reporter is indeed seen, so long as two conditions are met - the insert has to be in the intracellular domain of Notch, and the extracellular ligand Delta has to be present. Equivalent unpublished experiments in vertebrate cells by D. Baker and D. Ish-Horowicz give similar results (see ref. 9 for meeting report). Evidently, the intracellular part of Notch is launched directly from the membrane into the nucleus in response to the external signal.

How does binding of Delta (or of the related ligand, Serrate) trigger the cleavage of Notch? Nobody knows. But, according to unpublished findings by A. Israel and colleagues ${ }^{9}$, the final cut that unleashes NICD is the last in a series of three, the first two cuts being made in the extracellular domain. Once the bulk of the extracellular domain has been pruned away, the final intracellular cleavage follows automatically, regardless of the presence of ligand.

This unfinished story has a tantalizing twist. The intracellular cleavage that Schroeter et al. have identified occurs very close to, or even in, the transmembrane domain of the Notch protein. In this it resembles the intracellular cleavage of another, more sinister transmembrane molecule — the $\beta$-amyloid precursor protein, implicated in Alzheimer's disease. The most frequent genetic cause of familial early-onset Alzheimer's disease lies in missense mutations of the presenilin-1 gene PS1. Presenilin-1 is a membrane protein that is thought to govern the precise location at which the $\beta$-amyloid precursor protein is cleaved $^{10}$. The same cleavage process is probably also involved in the pathogenesis of those more common forms of Alzheimer's disease that do not arise from an inherited mutation. Genetic evidence from C. elegans and knockout mice indicates that presenilin1 is also a component of the Notch signalling pathway $^{11}$. So, as Struhl and Adachi ${ }^{2}$ and other Notch-watchers have noticed, presenilin- 1 is a candidate to be part of the mechanism that makes the cut that releases NICD. The same molecule that helps to endow us with a proper complement of neurons during our gestation may help to rob us of them in our dotage.
Julian Lewis is at the Imperial Cancer Research Fund, PO Box 123, Lincoln's Inn Fields, London WC2A 3PX, UK.

e-mail: j.lewis@icrficnet.uk

1. Schroeter, E. H., Kisslinger, J. A. \& Kopan, R. Nature 393, 382-386 (1998).

2. Struhl, G. \& Adachi, A. Cell 93, 649-660 (1998).

3. Lecourtois, M. \& Schweisguth, F. Curr. Biol. (in the press).

4. Lewis, J. Curr. Opin. Neurobiol. 6, 3-10 (1996).

5. Fortini, M. E. \& Artavanis-Tsakonas, S. Cell 79, 273-282 (1994).

6. Lieber, T., Kidd, S., Alcamo, E., Corbin, V. \& Young, M. W. Genes Dev. 7, 1949-1965 (1993).

Kopan, R., Schroeter, E. H., Nye, J. S. \& Weintraub, H. Proc.

Natl Acad. Sci. USA 93, 1683-1688 (1996).

8. Jarriault, S. et al. Nature 377, 355-358 (1995).

9. Bray, S. Cell 93, 499-503 (1998).

10. De Strooper, B. et al. Nature 391, 387-390 (1998).

11. Wong, P. C. et al. Nature 387, 288-292 (1997).

\title{
Ordering entropy
}

\section{Henk N. W. Lekkerkerker and Alain Stroobants}

T he study of colloidal systems has revealed many puzzles. To that list can now be added further examples, as presented in the paper by Adams and colleagues that appears on page 349 of this issue ${ }^{1}$. The authors have looked at mixtures of colloidal rods and spheres acting as 'hard' particles. Depending on the experimental conditions, the ensuing behaviour of the mixture can vary from, for instance, separation into phases in which the rods are or are not concentrated, and into alternating layers of rods and spheres.

Colloidal systems consist of small particles of one type of material in a continuous matrix of another type. They can be solid in liquid, like paint; liquid in liquid, like milk; and solid in gas, as in an aerosol. Simple yet brilliant experiments, carried out at the beginning of this century by Jean Perrin,

\section{Evolutionary biology \\ Land-loving crabs}

Like the eight other species of land crab found on Jamaica, the bromeliad crab pictured here is exceptionally well-adapted to terrestrial life. But despite their similar success in making a go of life on land, the nine species show great morphological variation, making it difficult to establish their origins by traditional means. Are they all the descendants of a single marine ancestor; did they evolve from several such ancestors; or are some more closely related to freshwater species? Elsewhere in this issue (Nature 393, 363-365; 1998),

Christoph D. Schubart and colleagues provide the answer.

Schubart et al. examined sequences from two mitochondrial genes of 22 marine and terrestrial crab species. The resulting phylogenetic trees reveal that the Jamaican terrestrial crabs originated from a single showed that, from a statistical point of view, a dilute suspension of colloidal particles behaves in much the same way as an ideal gas.

Perrin's view of colloids as atoms remains alive and well. Analogues of the well-known states of atomic and molecular matter (gas, liquid, crystal, liquid crystal, alloy, glass) have been observed and studied in concentrated colloidal suspensions in which the particles are interacting. Perhaps the oldest and most widely known example of a colloidal crystal, although it is not always recognized as such, is the gem opal. Opals consist of regularly packed silica particles with lattice spacings of the order of optical wavelengths. The resulting optical interference effects are the cause of opal's strikingly beautiful colours.

Why, then, study the phase behaviour of colloidal systems if it simply mimics that of atomic and molecular systems? The main 
reason is that the interaction between colloidal particles can be tuned by modifying the surfaces of the particles and the properties of the matrix in which they are suspended. In addition, both naturally occurring and synthetic colloidal particles are available in a wide range of shapes and sizes.

Adams et al. have exploited both of these characteristic features of colloidal systems to produce striking examples of new and interesting phase behaviour in mixtures of rodlike virus particles and synthetic colloidal spheres. Depending on the concentrations of the colloidal rods and the colloidal spheres, and on the size ratios, Adams et al. observe bulk demixing into rod-rich and rod-poor phases, and microphase separation involving a variety of ordered phases. One such phase, which the authors call 'lamellar', consists of layers of rods alternating with layers of spheres. In another, metastable yet longlived filaments of this lamellar structure coexist with either a nematic or a smectic phase. Finally, Adams et al. find experimental evidence for a highly ordered 'columnar' phase in which the spheres are packed in a two-dimensional array of columns orientated perpendicular to the rods.

At first sight one might think that such rich and subtle phase behaviour demands complex interactions between the particles. However, the experimental conditions are such that simple short-range repulsive interactions appear to be dominant. So, as the authors themselves say, entropy must be the driving force behind the phase transitions that they observe.

Entropy as a source of ordering might seem counterintuitive. As early as 1949, however, a seminal paper by Onsager showed unambiguously that, upon becoming concentrated, an isotropic fluid of thin, hard rods must undergo a transition to a nematic phase in which the rods have a preferred orientation. Onsager's argument was that, at sufficiently high concentration, the loss of entropy associated with the orientational ordering is more than compensated for by the gain in entropy associated with the increase in free volume in the orientationally ordered state. To understand this, one has to realize that (nearly) parallel rods take up less space than do randomly orientated rods, thus leaving more space to move in. Crystallization in an assembly of hard spheres, which was first subject to speculation by Kirkwood in 1939, and then persuasively exhibited by computer simulations in the late 1950s, might be understood on the basis of a similar argument: at sufficiently high density, the loss of entropy associated with positional ordering is more than compensated for by the gain in entropy associated with the increase in free volume.

Although the sphere case is geometrically simpler than the rod case, the arguments for entropy-driven crystallization in an assem- bly of hard spheres are less rigorous than those for the isotropic-nematic liquidcrystal phase transition in a hard rod system. Both types of transition have been experimentally observed in colloidal model systems. Furthermore, computer simulations have provided clear evidence that more highly ordered phases in systems of rods, such as the smectic phase and the crystalline phase, can also be produced by purely repulsive interactions - which again leaves entropy as the driving force.

The work of Adams et al. shows that combining rods and spheres leads to much richer phase-transition phenomena than those that occur in separate systems of spheres and rods. Once again, this phase behaviour must lead to a maximization of the entropy. Ultimately this means that the loss in orientational, positional and mixing entropy associated with the bulk and microphase separations must be more than compensated for by the gain in entropy associated with the increased free volume in the ordered structures that arises as a consequence of these phase transitions.

That this balance can give rise to remarkable results was already clear from the rich variety of alloy structures observed in mixtures of hard spheres of different sizes. Bartlett et al. ${ }^{2,3}$ observed the formation of $\mathrm{AB}_{2}$ and $\mathrm{AB}_{13}$ structures in a mixture of large and small colloids $\mathrm{A}$ and $\mathrm{B}$ with a diameter ratio of 0.58 . The computer simulations of Eldridge et al. ${ }^{4}$ confirm that these superlattice structures, which are highly complicated (the $\mathrm{AB}_{13}$ structure has 112 particles per unit cell), result from purely entropic effects.

So the free-volume effects in mixtures of spheres of different sizes are quite subtle. Not surprisingly, then, these effects have even more dramatic consequences in mixtures of rods and spheres (as it happens, microphase separation comparable to that seen in parts $b(1), b(2), c$ and d of Fig. 3 on page 350 has been observed in computer simulations, first in mixtures of rods of different length ${ }^{5}$ and later in rod-sphere mixtures $\left.{ }^{6}\right)$. These and the other novel structures observed by Adams et al. provide further challenges to understanding the way in which entropy produces such remarkable ordering.

Henk N. W. Lekkerkerker and Alain Stroobants are in the Van't Hoff Laboratory for Physical and Colloidal Chemistry, Debye Institute, Utrecht

University, Padualaan 8, 3584 CH Utrecht,

The Netherlands.

e-mail:h.n.w.lekkerkerker@chem.ruu.nl

1. Adams, M., Dogic, Z., Keller, S. L. \& Fraden, S. Nature 393, 349-352 (1998),

2. Bartlett, P., Ottewill, R. H. \& Pusey, P. N. J. Chem. 93, 1299-1312 (1990).

3. Bartlett, P., Ottewill, R. H. \& Pusey, P. N. Phys. Rev. Lett. 68, 3801-3804 (1992).

4. Eldridge, M. D., Madden, P. A. \& Frenkel, D. Nature 365, 35-37 (1993).

5. Stroobants, A. Phys. Rev. Lett. 69, 2388-2391 (1992).

6. Koda, T., Numajiri, M. \& Ikeda, S. J. Phys. Soc. Jpn 65, 3551-3556 (1996).

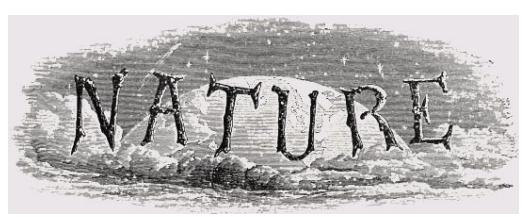

100 YEARS AGO

Undoubtedly, as the editor remarks in his preface to [this] work, there has been a great desire on the part of teachers of physiology in this country to obtain a complete text-book on their subject, written in English, somewhat similar to the classical Handbuch of Hermann. Prof. Schäfer, with the aid of some of the bestknown physiologists in Britain at the present day, has succeeded in bringing out a work which, if one may judge from the first volume, is destined to supply more or less completely the want that has been so long felt.-

Text-book of Physiology, edited by

\section{E. A. Schäfer, LL.D., F.R.S.}

From Nature 26 May 1898.

\section{YEARS AGO}

Physiology is a subject covering such a wide range of interests, and involving so many other branches of science, that teachers of it tend to have their own individual ways of approach. Most of them, after trying various ways of introduction to the subject, end up by considering, first, what that great teacher of physiology, Sir Michael Foster, called the 'master tissues' of the body, the muscles and the nerves.- Introduction to Physiology, by Prof. W. H. Newton.

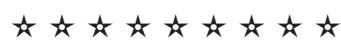

It is difficult to convey to this generation any adequate impression of the giant personalities that gave the Victorian era such brilliance and distinction. E. S. Beaven was one of them. Barley was his great life interest: he was the son of a barley grower, and son-in-law of a maltster whose business he entered as a youth; all his life was spent in handling barley and he knew it more intimately than any of his generation or this. But it was not only as a barley expert that he was known and respected: he was typical of his time, a sturdy, vigorous, forceful personality, outspoken, scathing in his denunciation of anything State-aided or other spoon-feeding agency; a stout believer in self-help, in some of his contemporaries, and in himself. From Nature 29 May 1948.

Many more abstracts like these can be found in A Bedside Nature: Genius and Eccentricity in Science, 1869-1953, a 266-page book edited by Walter Gratzer. Contact David Plant. e-mail: subscriptions@nature.com 